\title{
Effects of Picrorrhiza Rhizoma on Acute Inflammation in Mice
}

\author{
Hyeung Sik LEE ${ }^{1}$ and Sae Kwang Ku ${ }^{2,3 *}$ \\ ${ }^{1}$ Department of Herbal Biotechnology, Daegu Haany University, Gyeongsan 712-715, Republic of Korea \\ ${ }^{2}$ Department of Anatomy and Histology, College of Oriental Medicine, \\ Daegu Haany University, Gyeongsan 712-715, Republic of Korea \\ ${ }^{3}$ Development Team for the New Drug of Oriental Medicine (BK21 Program), \\ Daegu Haany University, Gyeongsan 712-715, Republic of Korea
}

(Received May 21, 2008; Accepted June 25, 2008)

\begin{abstract}
The effects of Picrorrhiza Rhizoma (PR) aqueous extracts were observed on xylene-induced acute inflammation. The xylene was topically applied 60 min after administration of 500, 250 and $125 \mathrm{mg} / \mathrm{kg}$ of PR extracts, and all animals were sacrificed 2 hrs after xylene application. The changes on ear weights, histolopathological analyses of ear were evaluated as compared with indomethacin and dexamethasone $15 \mathrm{mg} / \mathrm{kg}$ treated groups - well known anti-inflammatory agents. Xylene application resulted in marked increases in induced ear weights as compared with intact control ear. Severe vasodilation, edematous changes of ear skin and increase in the thickness of the ear tissues as acute inflammation were detected in xylene-treated control ears at histopathological observation. However, these xylene-induced acute inflammatory changes were dosedependently decreased by oral treatment of PR extracts. Therefore, it is concluded that PR extracts has favorable anti-inflammatory effects on xylene-applicated acute ear inflamed mice.
\end{abstract}

Keywords: Picrorrhiza Rhizoma, extracts, acute inflammation, xylene, histology, mouse

\section{INTRODUCTION}

Although inflammation has been regarded as an essential protective process preserving the integrity of organisms against various irritations like Physical damage, chemical substances, micro-organisms and other agents, the inflammatory response to several insults frequently leads to erroneous damage to normal tissues (Habashy et al., 2005).

A traditional Korean herbal medicine, Picrorrhiza Rhizoma (PR) is a dried root and stem of Picrorrhiza kurroa, and has been used as hepatoprotective agents such as jaundice. Until now, the nitric oxide scavenging activity (Jagetia and Baliga, 2004), cardioprotective effect (Senthil Kumar et al., 2001), anti-cancer effect (Jeena et al., 1999; Joy et al., 2000), anti-diabetic activity (Joy and Kuttan, 1999), anti-viral effect (Mehortra et al., 1990), hypolipidemic and hepatoprotective effects (Lee et al., 2006ab; Lee et al., 2008) of PR extracts have been evaluated. However, there are no experimental reports on the anti-

\footnotetext{
${ }^{*}$ Corresponding author

Tel: +82-53-819-1549, Fax: +82-53-819-1269

E-mail: gucci200@hanmail.net
}

inflammatory activities PR extracts as we know. Traditionally, water decoction of PR extracts has used as Korean medicine, the effects of aqueous PR extracts on the xylene-induced acute inflammation were investigated in the present study.

\section{MATERIALS AND METHODS}

\section{Animals and husbandry}

Sixty-three male ICR mice (6-week-old upon receipt; SLC, Japan) were used after acclimatization for 7 days. Animals were housed five or four per polycarbonate cage in a temperature $\left(20-25^{\circ} \mathrm{C}\right)$ and humidity $(40-45 \%)$ controlled room with a 12 hrs:12 hrs light:dark cycle. Feed (Samyang, Korea) and water were supplied ad libitum. All animals were fasted overnight before dosing and sacrifice (about $18 \mathrm{~h}$ with ad libitum access to water).

\section{Experimental design}

The PR was purchased from Cho-Heung Pharmaceutical Ind. Co. (Daegu, Korea) after confirming the morphology under microscopy. The prepared PR $(103 \mathrm{~g})$ was boiled in $2 \mathrm{I}$ of distilled water for $2 \mathrm{hrs}$ and filtrated. The fil- 
trate was decompressed using a rotary vacuum evaporator (Lab. Camp, Korea) and lyophilized in a programmable freeze dryer (IIShin Lab., Korea). Total acquired PR extracts was $26.4 \mathrm{~g}$ (yield 25.65\%). Powders of PR extracts were stored in a desiccator to protect from light and moisture. Indomethacin (Fluka, Switzerland) and dexamethasone (Sigma, USA) were used as reference drugs in this study. The animals were distributed into seven groups with 9 mice per group: intact control, xylene-treated control, 500, 250, and $125 \mathrm{mg} / \mathrm{kg}$ PR extracts dosing groups, $15 \mathrm{mg} / \mathrm{kg}$ indomethacin and dexamethasone dosing groups. PR extracts was orally administered once, and indomethacin and dexamethasone were administered intraperitoneally once. PR extracts was dissolved in distilled water, and indomethacin and dexamethasone were dissolved or suspended in saline.

\section{Induction of acute inflammation}

$1 \mathrm{hr}$ after oral administration with test substances, 0.03 $\mathrm{ml}$ of xylene (Merck, Germany) was topically applied to the anterior surface of the right ear. The left ear was considered a control.

\section{Ear weight measurement}

Two hours after topical application of xylene, all animals were sacrificed, and both ears were removed. Circular sections were taken using a cork borer with a 7-mm diameter and weighed as previously described (Kou et al., 2005). The absolute wet-weight of the induced ear was measured and then, the relative weights (\% of body weights) of the ears were calculated to reduce the differ- ences from individual body weights. In addition, the differences between the intact ear and induced ear were also calculated as follows:

Relative weight $(\%)=$ (absolute ear weight/body weight at sacrifice) $\times 100$

Differences in weights $(\mathrm{g})=$ induced ear weight - intact ear weight

\section{Histology and histomorphometry}

Induced ears were trimmed crosswise to include ear cartilage, and fixed in $10 \%$ neutral buffered formalin, then embedded in paraffin, sectioned $(3 \sim 4 \mu \mathrm{m})$ and stained with Hematoxylin \& Eosin stain. The histological profiles of the ears were observed as compared with intact or xylene-treated control. The thickness of the induced ear was calculated using automated image analysis (DMI300; DMI, Korea). The thicknesses from epidermis to dermis of induced ear skin (anterior skin thickness; $\mu \mathrm{m}$ ) and anterior to posterior ear (full ear thickness, $\mu \mathrm{m}$ ) were detected as histomorphometry on crossly trimmed ear specimens in regular corresponding regions in all induced ear samples.

\section{Statistical analyses}

Multiple comparison tests of the different dose groups were carried out. The variance homogeneity was examined using the Levene test. If the Levene test indicated no significant deviations from the variance homogeneity, the data obtain was analyzed using a one way ANOVA test followed by a least-significant difference multi-com-

Table I. Changes in ear weights in intact or xylene-applicated mice

\begin{tabular}{|c|c|c|c|c|c|c|}
\hline \multirow{2}{*}{ Group } & \multicolumn{3}{|c|}{ Absolute weight (mg) } & \multicolumn{3}{|c|}{ Relative Weight (\%) } \\
\hline & Intact ear & Induced ear & Difference & Intact ear & Induced ear & Difference \\
\hline \multicolumn{7}{|l|}{ Controls } \\
\hline Intact & $117.78 \pm 3.99$ & $121.67 \pm 5.55$ & $3.89 \pm 3.14$ & $0.42 \pm 0.01$ & $0.43 \pm 0.02$ & $0.01 \pm 0.01$ \\
\hline Xylene & $114.56 \pm 9.19$ & $154.78 \pm 6.65^{\star}$ & $40.22 \pm 11.39^{*}$ & $0.41 \pm 0.04$ & $0.56 \pm 0.02^{*}$ & $0.14 \pm 0.04^{*}$ \\
\hline \multicolumn{7}{|l|}{ References } \\
\hline Dexamethasone & $115.22 \pm 12.91$ & $124.89 \pm 12.44^{\#}$ & $9.67 \pm 10.76^{\#}$ & $0.42 \pm 0.04$ & $0.45 \pm 0.04^{\#}$ & $0.04 \pm 0.04^{\#}$ \\
\hline Indomethacin & $114.33 \pm 6.60$ & $132.67 \pm 9.41^{* *, \#}$ & $18.33 \pm 10.48^{\star, \#}$ & $0.41 \pm 0.02$ & $0.48 \pm 0.04^{*}, \#$ & $0.07 \pm 0.04^{*}, \#$ \\
\hline \multicolumn{7}{|l|}{ PR extracts } \\
\hline $500 \mathrm{mg} / \mathrm{kg}$ & $115.11 \pm 4.96$ & $130.89 \pm 10.13^{\star *}, \#$ & $15.78 \pm 12.12^{* *, \#}$ & $0.41 \pm 0.01$ & $0.47 \pm 0.04^{* *, \#}$ & $0.06 \pm 0.04^{*}, \#$ \\
\hline $250 \mathrm{mg} / \mathrm{kg}$ & $114.22 \pm 4.47$ & $135.44 \pm 6.17^{\star}, \#$ & $21.22 \pm 7.22^{*}, \#$ & $0.41 \pm 0.02$ & $0.49 \pm 0.03^{*}, \#$ & $0.08 \pm 0.03^{*}, \#$ \\
\hline $125 \mathrm{mg} / \mathrm{kg}$ & $115.33 \pm 3.64$ & $141.78 \pm 10.89^{\star}, \#$ & $26.44 \pm 13.21^{\star}, \# \#$ & $0.41 \pm 0.02$ & $0.51 \pm 0.05^{*}, \#$ & $0.10 \pm 0.05^{*}, \# \#$ \\
\hline
\end{tabular}

Mean \pm S.D. of nine mice; Relative weight $(\%)=($ absolute ear weight/body weight at sacrifice $) \times 100$; Differences=induced ear weight -intact ear weight; ${ }^{*} P<0.01$ and ${ }^{* *} P<0.05$ compared with. intact control; ${ }^{*} P<0.01$ and ${ }^{\# \#} P<0.05$ compared with xylene control by MW test. 
parison test to determine which pairs of group comparisons were significantly different. A non-parametric comparison test, Kruskal-Wallis $\mathrm{H}$ test was performed in the case of significant deviations from the variance homogeneity was observed at Levene test. When a significant difference was observed in the Kruskal-Wallis $\mathrm{H}$ test, the Mann-Whitney U-Wilcoxon Rank Sum W test was used to determine the specific pairs of group comparisons that were significantly different. The SPSS statistical software (Release 6.1.3, SPSS Inc., USA) was used and a $P$ value $<0.05$ was considered significant.
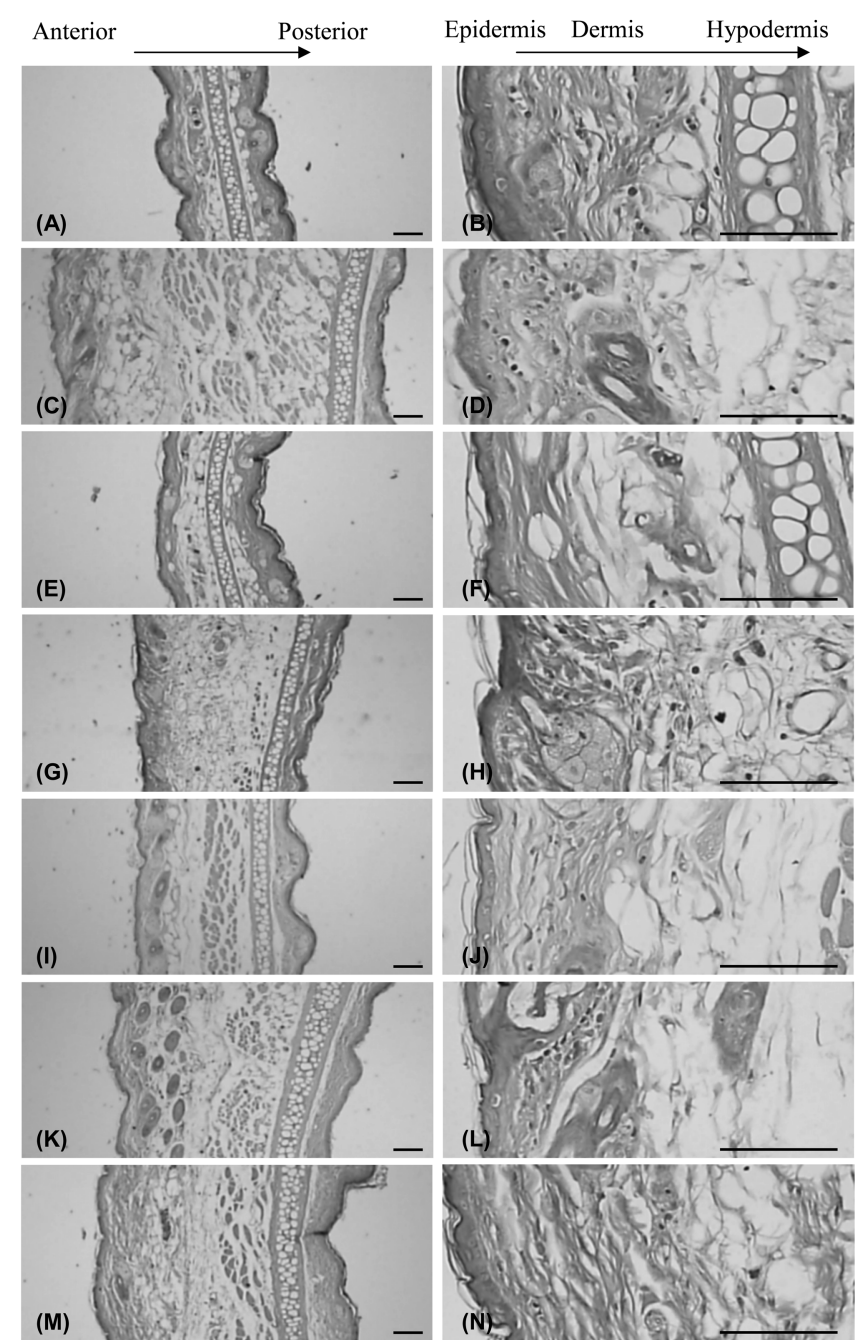

Fig. 1. Histological profiles of induced ear detected in intact control $(A, B)$, xylene control $(C, D)$, dexamethasone $(E, F)$, indomethacin $(\mathrm{G}, \mathrm{H}), 500(\mathrm{I}, \mathrm{J}), 250(\mathrm{~K}, \mathrm{~L})$ and $125(\mathrm{M}, \mathrm{N})$ of PR extracts-administered groups.

Note the classic histological profile of acute inflammation as severe vasodilation and edematous changes of skin were detected in xylene control. However, these inflammation indicators were dramatically decreased in all dosing groups tested. All Hematoxylin \& Eosin staining, Scale bars $=160 \mu \mathrm{m}$.

\section{RESULTS}

\section{Changes in ear weights}

Significant $(P<0.01)$ increases in the weights of the induced ear were detected in xylene-treated control as compared with intact control and, accordingly, the differences between intact and induced ear were also significantly $(P<0.01)$ increased regardless of absolute and relative weights. However, ear weights and the differences between intact and induced ear of all administered groups were significantly $(P<0.01$ or $P<0.05)$ decreased as compared with xylene-treated control, respectively (Table I).

\section{Histological analyses of induced ear}

Severe vasodilation and edematous changes of ear skin, as acute inflammation, was detected in xylenetreated control at histopathological observations. However, these histological indicators of acute inflammation were markedly and dose-dependently decreased in all three different dosages of PR extracts administered groups as compared with xylene-treated control (Fig. 1). Histomorphometrically, both anterior skin and full ear thicknesses were significantly $(P<0.01)$ increased in xylene-treated control as compared with intact control, but these increases in ear thickness induced by xylene applications were also significantly $(P<0.01)$ and dose-dependently inhibited by treatment of all three different dosages of PR extracts like two references (Table II).

Table II. Changes on the histomorphometry analyses of induced ear in intact or xylene-applicated mice

\begin{tabular}{lcc}
\hline \multicolumn{1}{c}{ Group } & $\begin{array}{c}\text { Full ear thickness } \\
(\mu \mathrm{m})\end{array}$ & $\begin{array}{c}\text { Anterior skin } \\
\text { thickness }(\mu \mathrm{m})\end{array}$ \\
\hline $\begin{array}{l}\text { Controls } \\
\text { Intact }\end{array}$ & $766.77 \pm 127.71$ & $324.39 \pm 94.26$ \\
Xylene & $1916.07 \pm 147.05^{*}$ & $1495.32 \pm 166.73^{*}$ \\
References & & \\
Dexamethasone & $763.84 \pm 103.99^{\#}$ & $328.82 \pm 71.95^{\#}$ \\
Indomethacin & $1059.19 \pm 221.11^{*, \#}$ & $739.26 \pm 185.30^{*, \#}$ \\
PR extracts & & \\
$500 \mathrm{mg} / \mathrm{kg}$ & $1007.32 \pm 136.44^{*, \#}$ & $690.09 \pm 151.01^{*, \#}$ \\
$250 \mathrm{mg} / \mathrm{kg}$ & $1341.77 \pm 222.92^{*, \#}$ & $1081.21 \pm 239.93^{*, \#}$ \\
$125 \mathrm{mg} / \mathrm{kg}$ & $1641.45 \pm 176.29^{*}, \#$ & $1166.73 \pm 163.32^{*, \#}$ \\
\hline
\end{tabular}

Mean \pm S.D. of nine mice; Anterior skin thickness=thicknesses from epidermis to dermis of induced ear skin; Full ear thickness=thicknesses from anterior to posterior ear of induced ear, ${ }^{*} P<0.01$ compared with intact control; ${ }^{\#} P<0.01$ and ${ }^{\# \#} P<$ 0.05 compared with xylene control. 


\section{DISCUSSION}

It is generally known that acute inflammation was easily induced by topical application of xylene on the ear skin, and the anti-inflammatory effect of a drug has been evaluated based on the changes of ear weight and histopathology (Hosseinzadeh et al., 2003; Kou et al., 2005). In the present study, the effects of PR extracts on xylene-induced acute inflammation were observed as compared with indomethacin (cyclooxygenase inhibitors) and dexamethasone (glucocorticoid), frequently used as references as development of the new anti-inflammatory drugs (Hosseinzadeh and Younsei 2002; Ramprasath et al., 2006).

Marked increases of ear weights (Al-Majed et al., 2003; Ojewole 2005), and severe vasodilation and edematous changes of ear skin at histopathological observation (De La Puerta et al., 1996; Kou et al., 2003; Rotelli et al., 2003; Kim et al., 2007) were detected as signs of acute inflammation after topical application of xylene in the present study. However, ear weight increases and histopathological changes were dose-dependently decreased after pretreatment with three different dosages of PR extracts in the present study. This inhibition was considered as direct evidence that the PR extracts used in this study has favorable effects on reducing the acute inflammatory response. These are considered as direct evidence that PR extracts inhibited the acute inflammatory response. Nitric oxide plays an important role in inflammation, and nitric oxide synthase inhibitors can reverse several inflammatory symptoms (Amin et al., 1995). Therefore, previously reported antioxidative effects of PR extracts (Jagetia and Baliga, 2004) were considered one of the major mechanisms of the anti-inflammatory effect of PR extracts detected in the present study. Based on the results, we therefore, conclude that the PR extracts has favorable effect on the reduction of the acute inflammatory response induced by xylene application in mice.

\section{REFERENCES}

Al-Majed, A. A., Khattab, M., Raza, M., Al-Shabanah, O. A. and Mostafa, A. M. (2003). Potentiation of diclofenac-induced antiinflammatory response by aminoguanidine in carrageenaninduced acute inflammation in rats: the role of nitric oxide. Inflamm. Res. 52, 378-382.

Amin, A. R., Vyas, P., Attur, M., Leszczynska-Piziak, J., Patel, I. R., Weissmann, G. and Abramson, S. B. (1995). The mode of action of aspirin-like drugs: effect on inducible nitric oxide synthase. Proc. Natl. Acad. Sci. USA 92, 7926-7930.

De La Puerta, R., Martinez, E., Bravo, L. and Ahumada, M. C. (1996). Effect of silymarin on different acute inflammation models and on leukocyte migration. J. Pharm. Pharmacol. 48, 968-970.
Habashy, R. R., Abdel-Naim, A. B., Khalifa, A. E. and Al-Azizi, M. M. (2005). Anti-inflammatory effects of jojoba liquid wax in experimental models. Pharmacol. Res. 51, 95-105.

Hosseinzadeh, H. and Younesi, H. M. (2002). Antinociceptive and anti-inflammatory effects of Crocus sativus L. stigma and petal extracts in mice. BMC Pharmacol. 2, 7-12.

Hosseinzadeh, H., Haddadkhodaparast, M. H. and Arash, A. R. (2003). Antinociceptive, antiinflammatory and acute toxicity effects of Salvia leriifolia Benth seed extract in mice and rats. Phytother. Res. 17, 422-425.

Jagetia, G. C. and Baliga, M. S. (2004). The evaluation of nitric oxide scavenging activity of certain Indian medicinal plants in vitro: a preliminary study. J. Med. Food 7, 343-348.

Jeena, K. J., Joy, K. L. and Kuttan, R. (1999). Effect of Emblica officinalis, Phyllanthus amarus and Picrorrhiza kurroa on Nnitrosodiethylamine induced hepatocarcinogenesis. Cancer Lett. 136, 11-16.

Joy, K. L. and Kuttan, R. (1999). Anti-diabetic activity of Picrorrhiza kurroa extract. J. Ethnopharmacol. 67, 143-148.

Joy, K. L., Rajeshkumar, N. V., Kuttan, G. and Kuttan, R. (2000). Effect of Picrorrhiza kurroa extract on transplanted tumours and chemical carcinogenesis in mice. J. Ethnopharmacol. 71, 261-266.

Kim, H. D., Cho, H. R., Moon, S. B., Shin, H. D., Yang, K. J., Park, B. R., Jang, H. J., Kim, L. S., Lee, H. S. and Ku, S. K. (2007). Effects of beta-glucan from Aureobasidium pullulans on acute inflammation in mice. Arch. Pharm. Res. 30, 323328.

Kou, J., Ni, Y., Li, N., Wang, J., Liu, L. and Jiang, Z. H. (2005). Analgesic and anti-inflammatory activities of total extract and individual fractions of Chinese medicinal ants Polyrhachis lamellidens. Biol. Pharm. Bull. 28, 176-180.

Lee, H. S., Ahn, H. C. and Ku, S. K. (2006a). Hypolipemic effect of water extracts of Picrorrhiza rhizoma in PX-407 induced hyperlipemic ICR mouse model with hepatoprotective effects: A prevention study. J. Ethnopharmacol. 105, 380-386.

Lee, H. S., Woo, S. J. and Ku, S. K. (2008). Hypolipemic and hepatoprotective effects of picrorrhiza rhizome in high fat diet supplied mice. A prevention study. Biomolecules \& Therapeutics 16, 46-53.

Lee, H. S., Yoo, C. B. and Ku, S. K. (2006b). Hypolipemic effect of water extracts of Picrorrhiza rhizoma in high fat diet treated mouse. Fitoterapia, 77, 579-584.

Mehrotra, R., Rawat, S., Kulshreshtha, D. K., Patnaik, G. K. and Dhawan, B. N. (1990). In vitro studies on the effect of certain natural products against hepatitis B virus. Indian J. Med. Res. 92, 133-138.

Ojewole, J. A. (2005). Antinociceptive, anti-inflammatory and antidiabetic effects of Bryophyllum pinnatum (Crassulaceae) leaf aqueous extract. J. Ethnopharmacol. 99, 13-19.

Ramprasath, V. R., Shanthi, P. and Sachdanandam, P. (2006). Immunomodulatory and anti-inflammatory effects of Semecarpus anacardium LINN. Nut milk extract in experimental inflammatory conditions. Biol. Pharm. Bull. 29, 693-700.

Rotelli, A. E., Guardia, T., Juarez, A. O., de la Rocha, N. E. and Pelzer, L. E. (2003). Comparative study of flavonoids in experimental models of inflammation. Pharmacol. Res. 48, 601-606.

Senthil Kumar, S. H., Anandan, R., Devaki, T. and Santhosh Kumar, M. (2001). Cardioprotective effects of Picrorrhiza kurroa against isoproterenol-induced myocardial stress in rats. Fitoterapia 72, 402-405. 